\title{
Investigation of plume dynamics during picosecond laser ablation of H13 steel using high-speed digital holography
}

\author{
Krste Pangovski $^{1}$ - Omonigho B. Otanocha ${ }^{2} \cdot$ Shan Zhong $^{2} \cdot$ Martin Sparkes $^{1} \cdot$

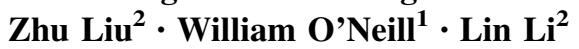

Received: 10 May 2016/ Accepted: 22 November 2016/Published online: 9 January 2017

(c) The Author(s) 2017. This article is published with open access at Springerlink.com

\begin{abstract}
Ablation of $\mathrm{H} 13$ tool steel using pulse packets with repetition rates of 400 and $1000 \mathrm{kHz}$ and pulse energies of 75 and $44 \mu \mathrm{J}$, respectively, is investigated. A drop in ablation efficiency (defined here as the depth per pulse or $\mu \mathrm{m} / \mu \mathrm{J}$ ) is shown to occur when using pulse energies of $E_{\text {pulse }}>44 \mu \mathrm{J}$, accompanied by a marked difference in crater morphology. A pulsed digital holographic system is applied to image the resulting plumes, showing a persistent plume in both cases. Holographic data are used to calculate the plume absorption and subsequently the fraction of pulse energy arriving at the surface after traversing the plume for different pulse arrival times. A significant proportion of the pulse energy is shown to be absorbed in the plume for $E_{\text {pulse }}>44 \mu \mathrm{J}$ for pulse arrival times corresponding to $>1 \mathrm{MHz}$ pulse repetition rate, shifting the interaction to a vapour-dominated ablation regime, an energetically costlier ablation mechanism.
\end{abstract}

\section{Introduction}

Recent advances in laser technology have furnished laser users with a variety of industrial short-pulse systems in the picosecond (ps) and femtosecond (fs) regimes, and are

Krste Pangovski

kp358@cam.ac.uk

1 Centre for Industrial Photonics, Institute for Manufacturing, University of Cambridge, Cambridge CB3 OFS, UK

2 Laser Processing Research Centre, School of Mechanical, Aerospace and Civil Engineering, The University of Manchester, Manchester M13 9PL, UK rapidly establishing themselves in industrial processes. Many authors have studied laser ablation in relation to laser-induced plumes [1-5] or material ejection mechanisms [6-9]; whilst many of these have made significant advances to our understanding of laser material interactions, available literature has almost exclusively dealt with single-pulse studies, be it from a theoretical or experimental standpoint [10-17].

Due to a lack of a general theoretical treatment, laser users resort to empirical parameter sweeps (pulse repetition rate, pulse energy, and focus position amongst others) to optimise a machining process. To avoid lengthy empirical studies, a variety of diagnostic approaches have been proposed. Techniques such as optical coherence tomography (OCT) [18], ultrasonic wave detectors based on probe beam deflection [19], Piezo-electric configurations [20], acoustic emission probes [21] as well as self-mixing interferometry, as demonstrated by Mezzapesa et al. [22], have also been applied. These, however, take no account of the plume dynamics.

The influence of a pre-formed plume on subsequent pulses in the picosecond regime can have a profound impact on the surface morphology $[23,24]$ through plume and plasma-enhanced surface heating. Onset of these phenomena leads to secondary energy coupling with molten ejecta, vapour, or plasma that can have a profound effect on the dynamics of the interaction and the final morphology of the affected site. The resulting plume forms a heat source at, and above, the processing zone that in turn interacts with the remainder of the incident and subsequent laser pulses. The main goal of this work is to elucidate the effect of pre-formed plumes on ablation of $\mathrm{H} 13$ steel under intense multi-pulse picosecond illumination, and determine the key parameters which influence plume development at the early stages of the process. 


\section{Materials and experimental procedure}

\subsection{Sample preparation}

Because of its industrial relevance, H13 tool steel was chosen as the processing material. H13 tool steel is an industrial-grade alloy containing carbon, manganese, silicon, chromium, phosphorus, molybdenum, and vanadium, as basic alloying elements. It is often used as a material in dies and moulds because of its favourable thermal and mechanical properties. The composition of $\mathrm{H} 13$ steel used is shown in Table 1. Test specimens were cut to size $(20 \mathrm{~mm} \times 25 \mathrm{~mm} \times 2 \mathrm{~mm})$ using a wire electro-discharge machine (EDM). The specimens were then shot blasted with fine grits, to produce even surfaces $\left(R_{a}=1.3 ; R_{\mathrm{p}}=6.6 ; R_{q}=1.7 ;\right.$ and $\left.R_{z}=14.7 \mu \mathrm{m}\right)$ devoid of surface impurities, and thereafter cleaned with compressed air to remove dust.

\subsection{Materials processing}

An Edgewave picosecond laser (model: QX-SN-0416) was used. The laser is a diode-pumped system (oscillator: $\mathrm{Nd}: Y V O 3)$ with an optical emission wavelength of $1064 \mathrm{~nm}$. The system operates with a pulse duration of $10 \mathrm{ps}$ and a repetition rate of $102 \mathrm{kHz}-19.2 \mathrm{MHz}$. The laser can reach an average output power up to $100 \mathrm{~W}$, with pulse energy up to $1 \mathrm{~mJ}$ and maximum peak power of $100 \mathrm{MW}$. The beam quality factor $M^{2}=1.2$, and pulse energy stability has $<4 \% \mathrm{rms}$ variation. To simulate a real industrial process, energy was delivered to the sample in pulse packets containing seven pulses each. Two pulse packet types are used in this study, shown in Table 2 . The choice of only seven pulses per packet was informed by the need to minimise thermal build-up and incubation effects in the substrate.

Post-process depth analysis was accomplished using a white-light interferometer, whilst surface morphology was examined using an SEM. Plume dynamics were studied using a high-speed digital holographic imaging system.

\section{High-speed digital holographic imaging system}

Dynamical material response was studied using a digital holographic imaging system, Fig. 1, previously demonstrated in $[23,25,26]$. The imaging laser is a TEEM

Table 1 Chemical composition of H13 tool steel (\%)

\begin{tabular}{lllllll}
\hline $\mathrm{C}$ & $\mathrm{Si}$ & $\mathrm{Mn}$ & $\mathrm{Cr}$ & $\mathrm{Mo}$ & $\mathrm{V}$ & $\mathrm{P} \& \mathrm{~S}$ \\
\hline 0.380 & 0.990 & 0.360 & 5.070 & 1.260 & 0.914 & 0.019 \\
\hline
\end{tabular}

The remainder of the composition is iron
Table 2 Pulse packet types used in this study, with corresponding pulse energy $E_{\mathrm{p}}$, pulse repetition rate PRF, total energy in burst $E_{\mathrm{t}}$, and duration of the pulse packet $\tau_{\mathrm{t}}$

\begin{tabular}{llccc}
\hline Pulse packet (notation) & $E_{\mathrm{p}}(\mu \mathrm{J})$ & PRF $(\mathrm{Hz})$ & $E_{\mathrm{t}}(\mu \mathrm{J})$ & $\tau_{\mathrm{t}}(\mu \mathrm{s})$ \\
\hline$P 7_{400 \mathrm{kHz}}^{75 \mu \mathrm{J}}$ & 75 & 400 & 525 & 15 \\
$P 7_{1000 \mathrm{kHz}}^{44 \mu \mathrm{J}}$ & 44 & 1000 & 308 & 6 \\
\hline
\end{tabular}

photonics frequency-doubled $Q$-switched PowerChip series platform (PNP-M08010) operating at $\lambda=532 \mathrm{~nm}$. The nominal pulse duration of the system is $\tau_{L}=400 \mathrm{ps}$. A Stanford research systems digital pulse generator (DG353) is used to trigger a single pulse. The pulse from the imaging laser is first cleaned using a spatial filter to produce a spatial $\mathrm{TEM}_{00}$ Gaussian beam profile. It is then split using a polarising beam splitter (BS1) into the object and reference wave-carrier arms. The object arm is expanded using a beam expander and propagated through the laserinduced "event" at the sample (element $X$ ) and imaged directly onto the CCD chip using a $4 f$ system (lenses L1, $\mathrm{L} 2$, and aperture $A$ ). The aperture $(A)$ was adjusted to remove high spatial frequencies, which could not be resolved by the CCD.

The field of view is chosen depending on the event under observation and appropriate optics, L1 and L2, selected. A narrow bandpass filter $(\lambda=532 \mathrm{~nm}$, FWHM $=1 \mathrm{~nm}$ ) was used to suppress plasma emission and luminescence. The reference arm is launched into a singlemode fibre and delivered through to the second beam splitter (BS2) prior to reaching the surface of the camera CCD chip. The camera is a iDS imaging $\mu$ Eye 3.0 Camera, $1 / 2^{\prime \prime}$ CMOS sensor operated in global shutter mode, with $2.2 \mu \mathrm{m}$ pixels. The temporal resolution of the system is limited by the pulse duration of the imaging laser. The object arm includes an optical delay line to ensure that the total path length difference between the object and reference arms is less than the coherence length of the imaging pulse, $\simeq 10 \mathrm{~mm}$. A photodiode (PD2) was used to determine the temporal overlap between the two reference arms, and the optical delay line was adjusted until there was good temporal overlap between the reference and object wavefronts, and clear interference fringes can be observed in the recorded image. The intensity of the two arms was equalised using the polarising beam splitter (BS1) to maximise fringe contrast and dynamic range recorded by the $\mathrm{CCD}$ camera. The pattern observed by the camera is formed from interference of the light from the focal plane of L1 and the output of the reference carrier fibre, both of which originate at the same plane with respect to the CCD plane. A Stanford research systems digital signal generator (DG353 model) was used to trigger the processing laser pulse train and the holography system sequentially, thus 


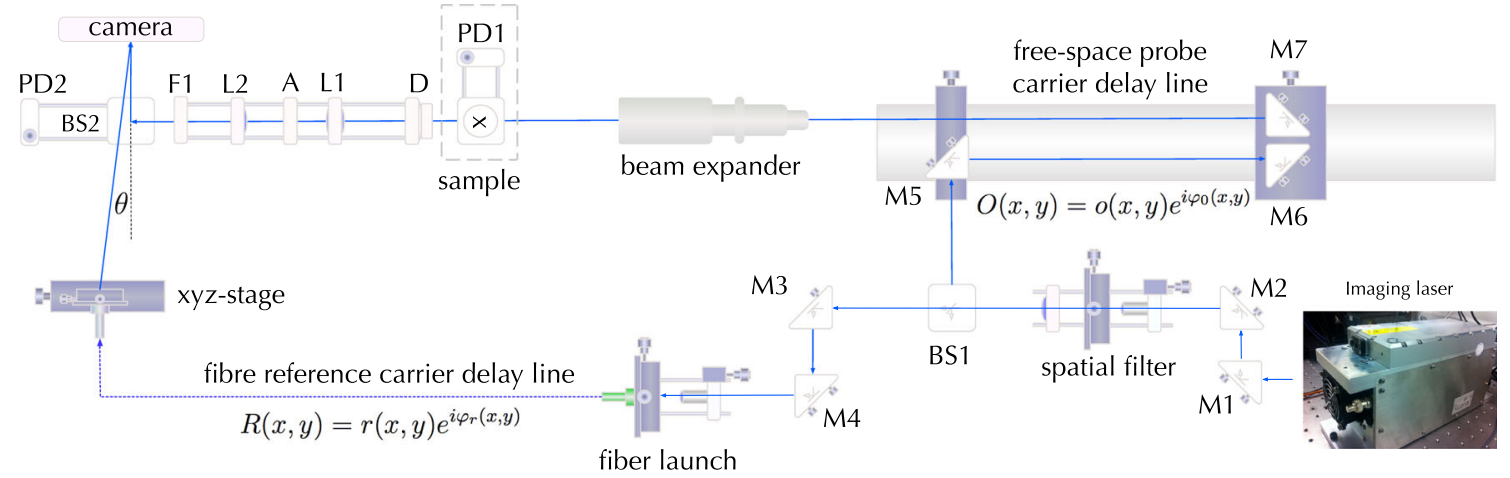

Fig. 1 A top-down schematic view of the digital holographic system

allowing imaging at any point in time during the processing up to $60 \mathrm{~s}$ in 1-ns steps.

During high-speed filming, first a reference hologram $I_{\mathrm{r}}$, is taken without activating the processing laser; this captures the ambient background and acts as a reference. Additional object holograms $I_{\mathrm{o}}(\Delta t)$ are acquired by triggering the camera at a time $\Delta t$ after the impact of the processing pulse. Precise $\Delta t$ measurements are ensured by integrated photodiodes (PD1 and PD2). A sequence of such images are acquired and reconstructed, using a MATLAB implemented algorithm $[23,25,26]$, to produce a highspeed movie of the event, allowing visualisation of the full refractive index history of the event.

All experiments were carried with the focal position of the scanner lens coincident with the sample surface, coincident with the bottom of the field of view of the camera to maximise the plume capture area. The processing laser is focused at the sample surface using a 80 -mm lens (into the page at element $X$ in Fig. 1).

\section{Results}

Machined site morphology is shown in Fig. 2. Site morphology for $P 7_{1000 \mathrm{kHz}}^{44 \mu \mathrm{J}}\left(7.0 \mathrm{~J} \mathrm{~cm}^{-2}\right)$ pulse bursts is characterised by two material removal regimes; a large ablated volume across the interacted zone and a smaller, deeper central region. The crater resulting from

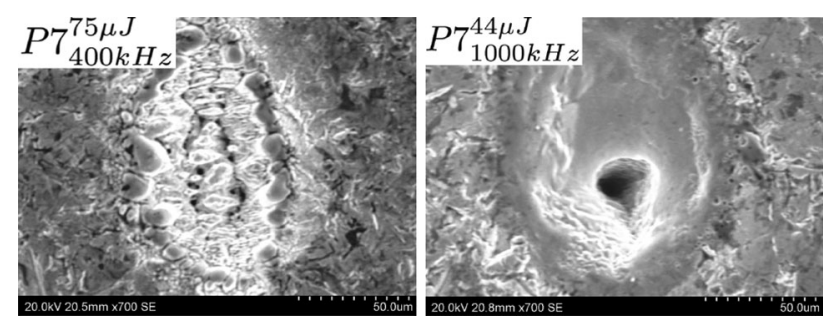

Fig. 2 SEM images of H13 ablated site morphology
$P 7_{400 \mathrm{kHz}}^{75 \mu \mathrm{J}}\left(11.9 \mathrm{~J} \mathrm{~cm}^{-2}\right)$ pulses is characterised by a rippled surface and is distinctly shallower than in the $P 7_{1000 \mathrm{kHz}}^{44 \mu \mathrm{J}}$ case.

Notably, although the total energy deposited during $P 7_{400 \mathrm{kHz}}^{75 \mu \mathrm{J}}$ machining was $217 \mu \mathrm{J}$ greater than for $P 7_{1000 \mathrm{kHz}}^{44 \mu \mathrm{J}}$ machining, the craters in the latter case are visibly deeper. Figure 3 shows the ablation rate characteristics of $\mathrm{H} 13$ under picosecond laser illumination. The interaction evolves as a gentle ablation regime, but beyond approximately $E_{\text {pulse }} \simeq 44 \mu \mathrm{J}$, there is a sudden drop-off in the ablation efficiency (defined here as the depth per pulse or $\mu \mathrm{m} / \mu \mathrm{J})$. This indicates a supra-surface interaction of the beam with the ejected material and a shift to an energetically costlier ablation process.

To explain the drop in crater depth, the resulting plumes were imaged the using the apparatus described in earlier sections. Plume morphologies, shown in Fig. 4, are displayed as refractive index maps. For $P 7_{400 \mathrm{kHz}}^{75 \mu \mathrm{J}}$ pulse bursts, the plume is characterised by a droplet shape. The plume starts to dissipate after $\simeq 2.4 \mu$ s at which time it begins to resemble the $P 7_{1000 \mathrm{kHz}}^{44 \mu \mathrm{J}}$ plume in size. Notably, the plume is separated from the surface indicating that some of the pulse has interacted with the plume. For $P 7_{1000 \mathrm{kHz}}^{44 \mu \mathrm{J}}$ pulse bursts, the plume is significantly smaller and forms near the

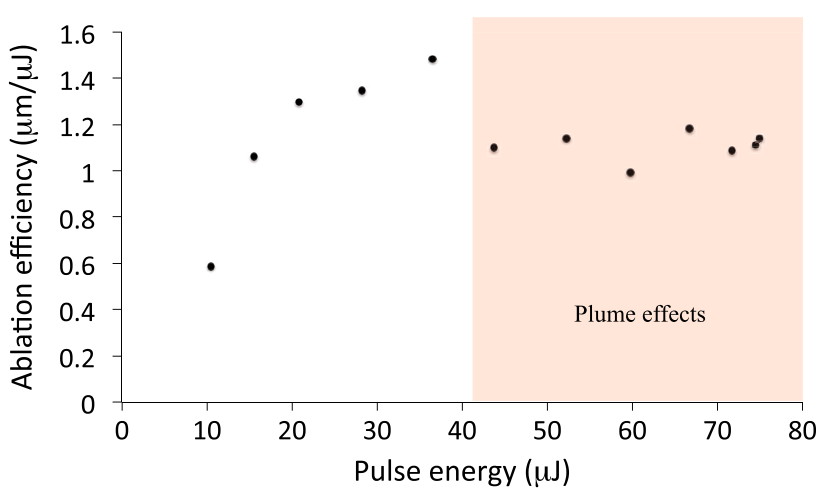

Fig. 3 Ablation efficiency characteristics of H13 under multi-pulse picosecond laser illumination, and onset of plume effects 


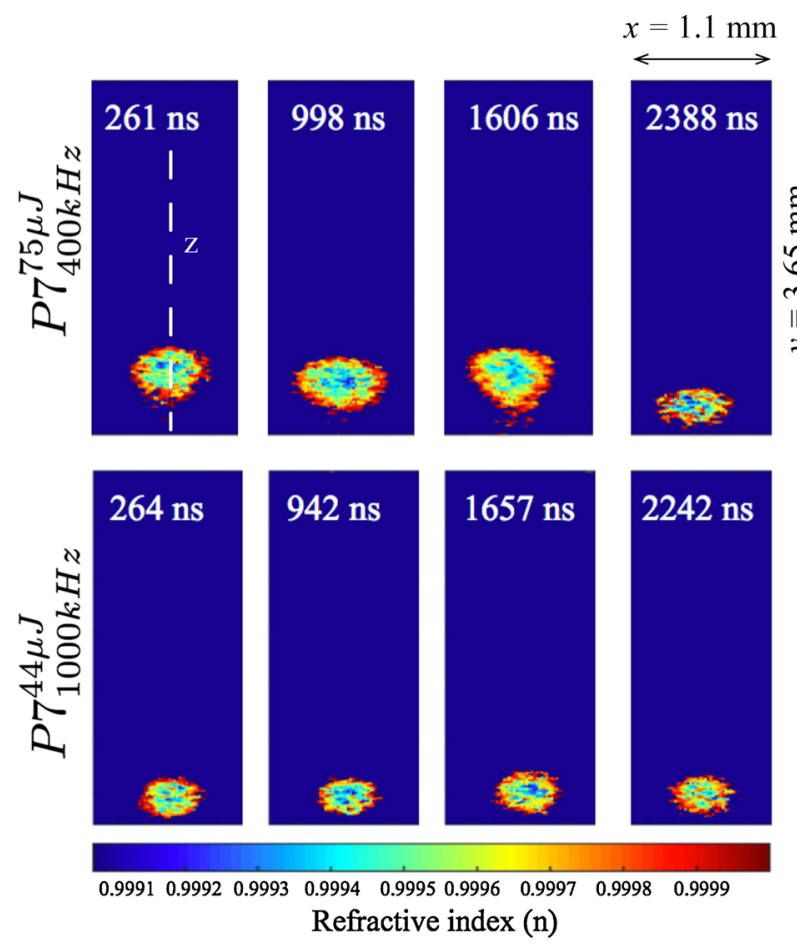

Fig. 4 Plume morphology for 44 and $75 \mu \mathrm{J}$ pulses. Time shown is relative to the last pulse in a burst. The field of view in each case is $x=1.1 \mathrm{~mm}$ by $y=3.65 \mathrm{~mm}$

surface and is roughly spherical in shape. It remains close to the surface throughout the interaction.

The refractive index maps are used to estimate the attenuation of pulses through the plume, and subsequently the amount of energy available at the sample surface. Holographic diagnostics of electron density do not require knowledge of absolute intensities and shapes from emission lines. The contribution of free electrons to the refractive index can be assumed to dominate if the probing wavelength is chosen far from any absorption lines $[3,27,28]$ :

$N_{\mathrm{e}} \simeq 10^{21} \lambda^{-2}\left(1-n^{2}\right)$

where the electron density is in cubic centimetres $\left(\mathrm{cm}^{-3}\right)$ and the wavelength is in micrometres. The temperature distribution is estimated by [29]:

$T_{\mathrm{p}}=\frac{T_{0}}{\left(\frac{n(x, y)-n_{0}}{n_{0}}\right)\left(\frac{3 P_{0} A+2 R_{\mathrm{c}} T_{0}}{3 P_{0} A}\right)+1}$

where $T_{0}$ is the ambient temperature $(300 \mathrm{~K})$, $P_{0}=101.3 \mathrm{kPa}\left(\mathrm{kg} \mathrm{m}^{-1} \mathrm{~s}^{-2}\right)$ and $R_{\mathrm{c}}=8.314 \mathrm{JK}^{-1} \mathrm{~mol}^{-1}$ is the molar gas constant and $A$ is the molar refractivity of air. If the inverse Bremsstrahlung (IB) mechanism is the dominant mechanism, the penetration depth of a pulse through the plasma - analogous to penetration in materials—can be defined as [20]:
$l_{\mathrm{IB}}=a_{\mathrm{c}} T_{\mathrm{e}}^{3 / 2}\left(N_{\mathrm{c}} / N_{\mathrm{e}}\right)^{2}$

where $T_{\mathrm{e}}$ is the electron temperature in electron volts $(\mathrm{eV})$, $N_{\mathrm{e}}$ is the electron density, from Eq. (1), and the $\lambda$-dependent critical plasma density $N_{\mathrm{c}}=10^{21} \lambda^{2} \mathrm{~cm}^{-3}$ in the case of $\lambda=1064 \mathrm{~nm}$ [20,30], where $\lambda$ is the laser wavelength in micrometres. The characteristic IB length is defined as $a_{\mathrm{c}}=10^{-16} Z \ln \Lambda$, for ions of $Z e$ electric charge where $e$ is the electron charge, $Z$ is the charge number, and $\ln \Lambda$ is the Coulomb logarithm [20,31], where $\Lambda \simeq 15$ is the plasma parameter [32], for many laser-produced plasmas.

When estimating the penetration depth of Eq. (3) we make the assumption that the plume temperature $T_{\mathrm{p}}$ from Eq. (2) and electron temperature $T_{\mathrm{e}}$ from Eq. (1) are in equilibrium. Once the penetration depth has been estimated, the total loss a laser pulse would experience by propagating a distance $z$ through the plume can be estimated by:

$I(z)=I_{0} \mathrm{e}^{-z / l_{\mathrm{IB}}}$

where $I_{0}$ is the incident intensity and $I(z)$ is the pulse intensity after propagating through a distance $z$ through the plume. The penetration depth $l_{\mathrm{IB}}$ changes along the plume (due to variations in $n, T_{\mathrm{p}}$ and $N_{\mathrm{e}}$ ) so this is calculated per pixel. Additionally, the assumption is made that the pulses are equivalent to delta functions.

These values were used to estimate the percentage of original energy remaining and available at the sample surface after propagating through the plume, shown in Fig. 5. Pulses in the $P 7_{400 \mathrm{kHz}}^{75 \mu \mathrm{J}}$ case are attenuated to $\simeq 55 \%$ of their original value if they arrive $<1 \mu$ s after the previous pulse impact. Conversely, for the same arrival time representing a $1-\mathrm{MHz}$ pulse repetition frequency, a pulse with $44 \mu \mathrm{J}$ of energy would only be attenuated to $\simeq 80 \%$ of the original value. In the case where the pulses arrive at $2.5 \mu \mathrm{s}$ (corresponding to a repetition rate of $400 \mathrm{kHz}$ ), the plume has dissipated significantly, and the pulses are attenuated to $\simeq 91 \%$ in both cases.

Several factors now explain the drop in crater depth. Firstly, considering the crater features particularly the rippled surface for the $P 7_{400 \mathrm{kHz}}^{75 \mu \mathrm{J}}$ case, and increase in plume size supports the case for a vapour-dominated regime which is an energetically costlier mechanism for material removal. Secondly, plume attenuation-related to vapour and plasma density-is more prominent for the $P 7_{400 \mathrm{kHz}}^{75 \mu \mathrm{J}}$ interaction when considering both the physical size and composition of the plume. Finally, as pulse energy is reduced, the interaction reverts to a melt-dominated regime, whereupon the total energy input is more dominant than any dynamic effects. The melt-dominated regime is confirmed by holographic imaging of the plume, using a particle detection algorithm [25], and shown in Fig. 6. 


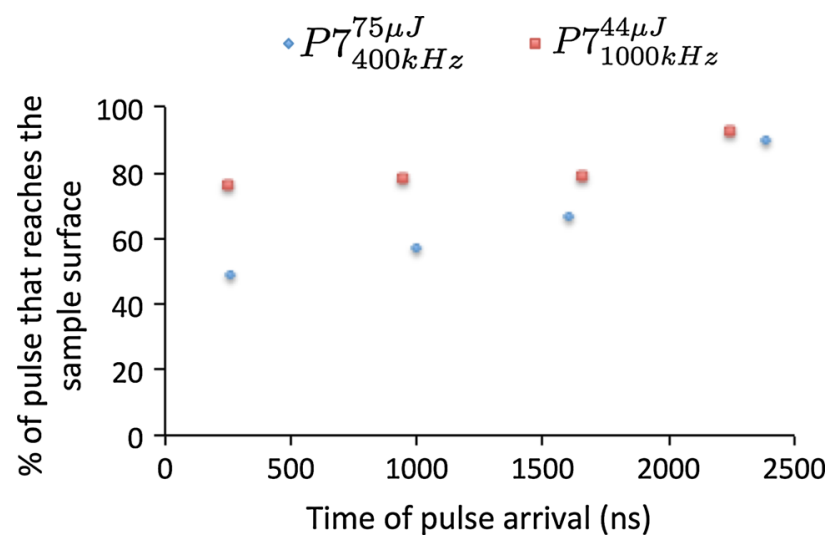

Fig. 5 Remaining energy in the pulse after propagating through different plumes

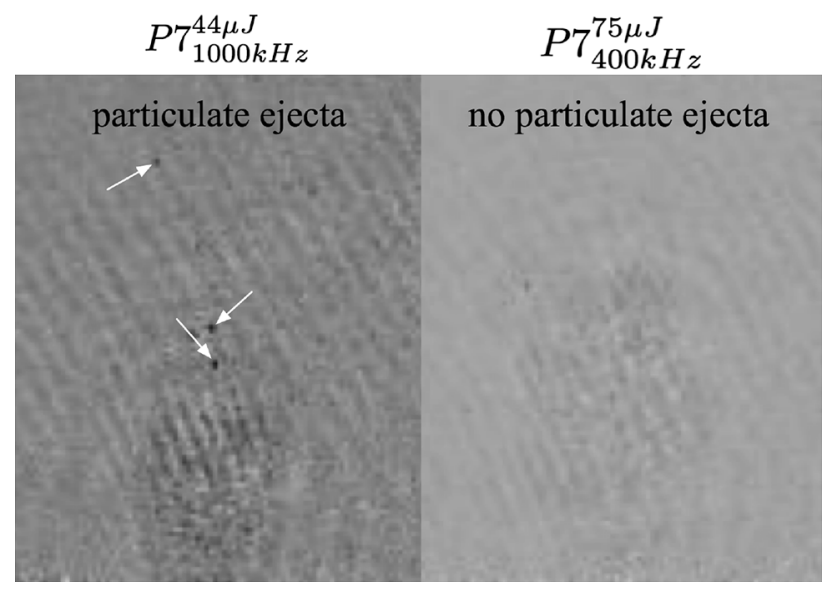

Fig. 6 Holographic particle detection showing particulate ejecta for $44 \mu \mathrm{J}$ pulses and no particles for $75 \mu \mathrm{J}$ pulses. Arrows indicate position of particle ejecta

For the $P 7_{1000 \mathrm{kHz}}^{44 \mu \mathrm{J}}$ case, molten particulate ejecta is clearly visible. In the case of $P 7_{400 \mathrm{kHz}}^{75 \mathrm{~J}}$ pulses, all the energy is used to vaporise the material and no ejecta is detected.

\section{Conclusions}

Digital holography has been applied in conjunction with traditional analysis techniques (SEM and white-light interferometry) to study the effect of pre-formed plumes on H13 steel under intense multi-pulse picosecond illumination. The decrease in ablation efficiency is shown to be the effect of an increased plume absorption and a concurrent shift to an energetically costlier, vapour-driven ablation mechanism.

High-energy pulses operating at reduced frequencies tend to produce a vapour-dominated interaction, which lead to increased laser absorption in the resulting plume. Lower energy pulses operating at high frequencies shift the interaction towards a melt-dominated regime partly by acting as a secondary heat source closer to the interaction zone, whilst simultaneously delivering a greater percentage of the available energy to the sample surface, contributing to thermal incubation in the bulk material. Notably, plume densities can remain high for several microseconds after pulse packet termination, and whilst the plume is still present above the interaction zone, absorptivity will drop until it fully dissipates.

In the $P 7_{400 \mathrm{kHz}}^{75 \mu \mathrm{J}}$ case, the total ablated material in the form of a vapour is greater, leading to higher absorptivity of the plume and a longer path length over which absorption takes place. Conversely, in the $P 7_{1000 \mathrm{kHz}}^{44 \mu \mathrm{J}}$ case, individual pulses contribute less vapour resulting in a smaller plume and a shorter path length over which subsequent pulses can be attenuated; this results in a slowly varying plume which remains above the interaction zone throughput the process that contributes thermal energy to the substrate leading to molten ejection.

The approach presented in this work could be applied in real industrial processes to optimise energy delivery strategy based on recorded holographic data, either by delivering subsequent pulses away from the geometric footprint of the plume, or by determining the time required for the plume to dissipate to an acceptable attenuation value.

Acknowledgements This work was collaboratively carried out under EPSRC Grant Number EP/K030884/1, as part of the EPSRC Centre for Innovative Manufacturing in Laser-based Production Processes. One of the authors acknowledges his PhD studentship by the Federal Government of Nigeria (TETFUND) in conjunction with the Federal University of Petroleum Resources Effurun (FUPRE).

Open Access This article is distributed under the terms of the Creative Commons Attribution 4.0 International License (http://crea tivecommons.org/licenses/by/4.0/), which permits unrestricted use, distribution, and reproduction in any medium, provided you give appropriate credit to the original author(s) and the source, provide a link to the Creative Commons license, and indicate if changes were made.

\section{References}

1. H. Schittenhelm, G. Callies, P. Berger, H. Hugel, Measurements of wavelength-dependent transmission in excimer laser-induced plasma plumes and their interpretation. J. Phys. D: Appl. Phys. 31(418), 418-427 (1998)

2. J.-F.Y. Gravel, D. Boudreau, Study by focused shadowgraphy of the effect of laser irradiance on laser-induced plasma formation and ablation rate in various gases. Spectrochim. Acta Part B: At. Spectrosc. 64(1), 56-66 (2009)

3. D. Breitling, H. Schittenhelm, P. Berger, F. Dausinger, H. Hügel, Shadowgraphic and interferometric investigations on Nd:YAG laser-induced vapor/plasma plumes for different processing wavelengths. Appl. Phys. A: Mater. Sci. Process. 69(7), S505S508 (1999) 
4. A.V. Bulgakov, N.M. Bulgakova, Dynamics of laser-induced plume expansion into an ambient gas during film deposition. Phys. D: Appl. Phys. 28(8), 1710-1718 (1995)

5. A. Bogaerts, Z. Chen, Effect of laser parameters on laser ablation and laser-induced plasma formation: a numerical modeling investigation. Spectrochim. Acta Part B: At. Spectrosc. 60(9-10), 1280-1307 (2005)

6. Q. Lu, S.S. Mao, X. Mao, R.E. Russo, Delayed phase explosion during high-power nanosecond laser ablation of silicon. Appl. Phys. Lett. 80(17), 3072 (2002)

7. Y. Zhou, B. Wu, S. Tao, A. Forsman, Y. Gao, Physical mechanism of silicon ablation with long nanosecond laser pulses at $1064 \mathrm{~nm}$ through time-resolved observation. Appl. Surf. Sci. 257(7), 2886-2890 (2011)

8. S. Tao, Y. Zhou, B. Wu, Y. Gao, Infrared long nanosecond laser pulse ablation of silicon: integrated two-dimensional modeling and time-resolved experimental study. Appl. Surf. Sci. 258(19), 7766-7773 (2012)

9. J.H. Yoo, S.H. Jeong, X.L. Mao, R. Greif, R.E. Russo, Evidence for phase-explosion and generation of large particles during high power nanosecond laser ablation of silicon. Appl. Phys. Lett. 76(6), 783 (2000)

10. S.T. Hendow, R. Romero, S.A. Shakir, P.T. Guerreiro, Percussion drilling of metals using bursts of nanosecond pulses. Opt. Express 19(11), 10221-10231 (2011)

11. J.M. Fishburn, M.J. Withford, D.W. Coutts, J.A. Piper, Study of the fluence dependent interplay between laser induced material removal mechanisms in metals: vaporization, melt displacement and melt ejection. Appl. Surf. Sci. 252(14), 5182-5188 (2006)

12. J. König, S. Nolte, A. Tünnermann, Plasma evolution during metal ablation with ultrashort laser pulses. Opt. Express 13(26), 10597-10607 (2005)

13. S.H. Jeong, R. Greif, R.E. Russo, Shock wave and material vapour plume propagation during excimer laser ablation of aluminium samples. J. Phys. D: Appl. Phys. 32, 2578 (1999)

14. R.E. Russo, X.L. Mao, H.C. Liu, J.H. Yoo, S.S. Mao, Timeresolved plasma diagnostics and mass removal during singlepulse laser ablation. Appl. Phys. A 894, 887-894 (1999)

15. E. Amer, P. Gren, A.F.H. Kaplan, M. Sjödahl, Impact of an extended source in laser ablation using pulsed digital holographic interferometry and modelling. Appl. Surf. Sci. 255(21), 8917-8925 (2009)

16. E. Amer, P. Gren, A.F.H. Kaplan, M. Sjödahl, M. El Shaer, Comparison of the laser ablation process on $\mathrm{Zn}$ and $\mathrm{Ti}$ using pulsed digital holographic interferometry. Appl. Surf. Sci. 256(14), 4633-4641 (2010)

17. V.V. Temnov, K. Sokolowski-Tinten, P. Zhou, D. von der Linde, Ultrafast imaging interferometry at femtosecond-laser-excited surfaces. J. Opt. Soc. Am. B 23(9), 1954 (2006)
18. P.J.L. Webster, M.S. Muller, J.M. Fraser, High speed in situ depth profiling of ultrafast micromachining. Opt. Express 15(23), 14967-14972 (2007)

19. S. Strgar, J. Mozina, An optodynamic determination of the depth of laser-drilled holes by the simultaneous detection of ultrasonic waves in the air and in the workpiece. Ultrasonics $40(1-8)$, 791-795 (2002)

20. M. Stafe, C. Negutu, I.M. Popescu, Real-time determination and controlof the laser-drilled holes depth. Shock Waves 14(1-2), 123-126 (2005)

21. M.G. Droubi, R.L. Reuben, G. White, Monitoring acoustic emission (AE) energy in slurry impingement using a new model for particle impact. Mech. Syst. Signal Process. 62, 415-430 (2015)

22. F.P. Mezzapesa, A. Ancona, T. Sibillano, F. De Lucia, M. Dabbicco, P.M. Lugarà, G. Scamarcio, High-resolution monitoring of the hole depth during ultrafast laser ablation drilling by diode laser self-mixing interferometry. Opt. Lett. 36(6), 822-824 (2011)

23. K. Pangovski, M. Sparkes, W. O'Neill, A holographic method for optimisation of laser-based production processes. Adv. Opt. Technol. 5(2), 177-186 (2016)

24. S.S. Mao, X. Mao, R. Greif, R.E. Russo, Influence of preformed shock wave on the development of picosecond laser ablation plasma. J. Appl. Phys. 89(7), 4096 (2001)

25. K. Pangovski, M. Sparkes, A. Cockburn, W. O'Neill, D. Richardson, Control of material transport through pulse shape manipulation-a development toward designer pulses. IEEE J. Sel. Top. Quantum Electron. 20(5), 51-63 (2014)

26. A.G. Demir, K. Pangovski, W. O’Neill, B. Previtali, Investigation of pulse shape characteristics on the laser ablation dynamics of TiN coatings in the ns regime. J. Phys. D: Appl. Phys. 48(23), $235202(2015)$

27. H.R. Griem, Plasma Spectroscopy (McGraw-Hill, New York, 1964)

28. S. Amoruso, R. Bruzzese, N. Spinell, R. Velotta, Characterization of laser-ablation plasmas. J. Phys. B: At. Mol. Opt. Phys. 32(14), R131-R163 (1999)

29. S. Sharma, G. Sheoran, C. Shakher, Digital holographic interferometry for measurement of temperature in axisymmetric flames. Appl. Opt. 51(16), 3228-3235 (2012)

30. E. Amer, P. Gren, M. Sjödahl, Laser-ablation-induced refractive index fields studied using pulsed digital holographic interferometry. Opt. Lasers Eng. 47(7-8), 793-799 (2009)

31. T.W. Murray, J.W. Wagner, Laser generation of acoustic waves in the ablative regime. J. Appl. Phys. 85(4), 2031 (1999)

32. J.D. Huba, NRL Plasma Formulary (The office of naval research, Washington DC, 2009) 